\title{
The Analysis and Simulation of Remote Underwater Communication Method Based on Airgun Source
}

\author{
Xiaoliang Zhang ${ }^{1,}$, song $\mathrm{Xu}^{2}$, Kejia $\mathrm{Yi}^{1}$ \\ ${ }^{1}$ Science and Technology on Underwater Acoustic Antagonizing Laboratory, China \\ ${ }^{2}$ Systems Engineering Research Institute of CSSC, China \\ azxl.seri@163.com
}

Keywords: airgun source; remote control; pulse coding; underwater acoustic communication

\begin{abstract}
This paper presents a long-distance underwater acoustic communication remote control method in which airgun is used as the source. This method expands the range of underwater remote and supports underwater robots to work together. The pulse signal (produced by airgun) source level, frequency and consistency of multiple transmission analyze the feasibility of airgun as the underwater acoustic communication signal source. Based on instruction-level remote control information transmission needs, proposes delay pulse coding method and signal detection and identification method. Under different hydrological environmental conditions, do simulation and analysis, provides an alternative technological approach for long-distance underwater acoustic communication remote control.
\end{abstract}

\section{Introduction}

Airgun uses the underwater instant release of high pressure air stored in chamber to produce intense sound waves. Because of its excellent low-power features, it has been widely used in the field of marine geological survey, oil exploration, seismic wave research. In this paper, the airgun is used as underwater acoustic communication source, make use of the characteristics that low-frequency high-power pulse signal (generated by the underwater instant release of high pressure air)can transmit over a long distance, use the pulse delay coding, match filtering and dual- $\alpha$ filtering detection method to solve the transient pulse signal coding, detection and identification issues, realize long-distance underwater remote communication. The remote distance is expected to reach hundreds of kilometers. Long-distance underwater robots operational requirements are satisfied.

\section{The Pulse Signal Encoding Method}

The existing references have confirmed that the airgun source waveform consistency is very high under the same condition of acoustic source structure and trigger. There's much better consistency between pulse signals which generated through multiple trigger. The statistical correlation coefficient of multiple emission of airgun acoustic source can be up to 0.996 [1]. Thus, the characteristic of acoustic impulse signal which generated by airgun acoustic source that possess controllability and repeatability. The acoustic impulse signal is used for instruction level underwater acoustic communication through coding for continuous trigger pulse sequence adjusting the variable parameters (Triggered delay time, Cabin pressure).

While the system implementing the remote low-speed underwater acoustic communication, we only send instruction-level information such as "start”, “operating mode n”, "standby”, etc. There's less demanding on the communication bandwidth and complex coded modulation communication system. For instruction encoding, we can adopt pulse delay coding. The information can be expressed as multiple pulses interval.

As we all known, the most suitable form of channel coding should be transmitted in the form of white noise. In other words, the symbol 0 and the symbol 1 should not continuously connect in line with the form of white noise. So the first step of optimizing the code is to get rid of the instruction 
code sequence which there is a large number of symbols in a row of 1 and symbols in a row of 0 . These sequences must be weeded out. Then, the main criterion to select the set of coding command is followed by a low correlation and the large enough code distance of sequence. The method of generating pseudorandom sequence (such as the Gold sequences) may be used to generate the coding sequence. For example, the duration of coding is $10 \mathrm{~s}$, the coding interval is $1 \mathrm{~s}$. It would give priority to the one with minimum correlation and maximum symbol spacing. And 0 indicates no pulse, 1 indicates that there's pulse existing. We would control the delay interval of pulse emission. The set of command signal which in Table 1 below.

Table 1 Set of coding command

\begin{tabular}{|l|l|}
\hline Set of command & Coding sequence \\
\hline Start & 10101000001 \\
\hline Operating mode 1 & 10000100101 \\
\hline$\ldots \ldots$ & $\ldots \ldots$ \\
\hline Operating mode $\mathrm{n}$ & 10100100001 \\
\hline standby & 10000010101 \\
\hline
\end{tabular}

\section{Signal Detection and Identification Method}

The long-distance transmission, the reflection between surface and floor and varied influences lead to sever signal distortion and multi-path interference to underwater acoustic pulse signal that generated by airgun acoustic source. The detection and identification for coded pulse signal is the most difficult part of the remote underwater acoustic communication. A reverse design methods can be used to build a propagation model, analyze respectively pulse width, pulse code and pulse distortion after long distance transmission. Through simulation and actual measurement, transmission channel would build a relationship with pulse broadening and time delay interval. Take the receiving end can accurately and reliably detect and recognize communication signals as basic requirements, it infers the transmit pulse parameters set at the transmitting terminal. According to the practical application, we can adjust the pulse width and the pulse interval of the transmission signal, to ensure that the receiving end can receive a communication signal with good consistency. We can adopt match-filtering, double- $\alpha$ filter detection [2,3], correlation detection [4], etc. to detect of communication signal. To carry out the detection of weak pulse signal which through distant propagation.

This paper used a detection method combining match-filtering together with double- $\alpha$ filtering. The match filter is an optimal linear filter in the case that certain input signals with the white noise. The resulting output is of the maximum SNR (SNR: signal to noise ratio). It can effectively improve the SNR by using a match filter. The output signal of match filter is judged by double- $\alpha$ filter. And the pulse position is accurately determined. Furthermore, according to the characteristics of the double- $\alpha$ filter, it not only can track the signal but also can smooth it. When $\alpha$ tends to 0 , it shows tracking feature in main. When $\alpha$ tends to 1 , it shows smoothing filter feature in main. Respectively using tracking and smoothing characteristics when both two filters working. According to channel conditions, it set $\mathrm{M}$ with a suitable magnitude as judgment threshold. When $y_{1}>M y_{2}$, the arrival of the synchronization signal is determined. Then it searches in a range around the position of the synchronization signal to ensure the exact position of the pulse peak.

The matching method of instruction set is used in communication signal recognition. According to the needs of communication, parameters (such as the pulse width, frequency range, the pulse interval, pulse number) of the communication signal are agreed well in advance. Establishing a communication command set and matching the received acoustic signal at the receiver. 


\section{Simulation and Verification}

The methods was simulated and analyzed with the main simulation conditions and parameters setting as follows:

(1)The parameters of ocean environment

The sea depth $200 \mathrm{~m}$, sound speed $1500 \mathrm{~m} / \mathrm{s}$. Constant gradient velocity profile, positive gradient velocity profile and negative gradient velocity profile were simulated and analyzed. The article lists only the simulation results of constant gradient velocity profile.

(2)The parameters of source and receiving hydrophone

The depth of airgun source $10 \mathrm{~m}$, SL (SL: sound level) $210 \mathrm{~dB}$, the depth of receiving hydrophone $5 \mathrm{~m}$, the communication distance $200 \mathrm{~km}$.

The waveform of airgun source pulse, channel impulse response and the loss of transmission simulated are illustrated in Fig. 1.
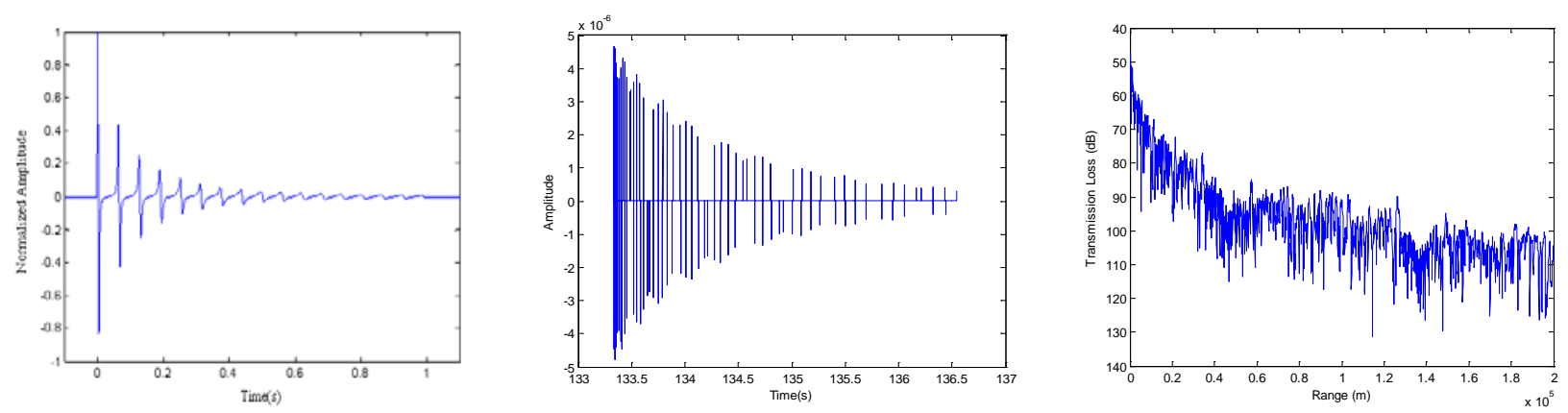

Fig. 1 Channel impulse response and transmission loss of constant gradient velocity profile

Select "1010101010" as the communication instruction sequence. The waveform of received signal which cross through the acoustic channel, matched filter output, double $\alpha$ filter output, and the estimation of the pulse delay are illustrated in Fig. 2.

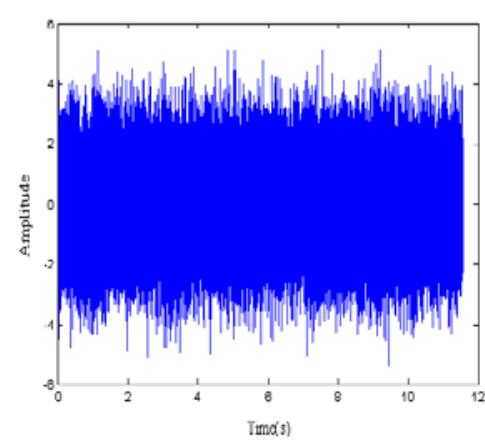

(a) Received signal waveform

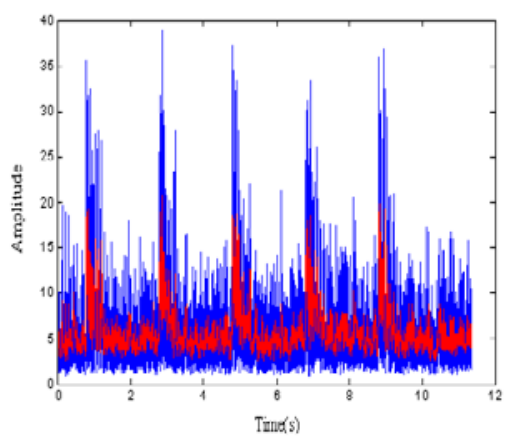

(b) Double $\alpha$ filter output

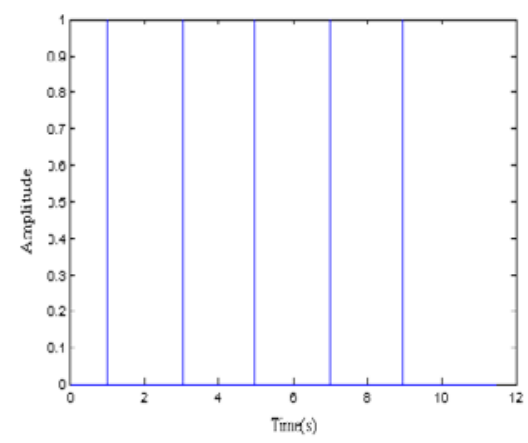

(d) The time delay estimation result

Fig 2 The result of simulation processing

After the position purification process, the time delay information of pulse can be accurately estimated and agrees well with the communication instruction sequence. The simulation result means that the long-distance underwater acoustic communication method proposed in this paper is effective.

\section{Analysis of Performance Optimization}

The detection and estimation of underwater acoustic signal has greater impact by the received SNR. This paper has carried on the simulation and statistics for the BER (BER: bit error rate) under different received SNR, and the result is illustrated in Fig. 3. There are two ways to improve the received SNR. One is to improve the source level as far as possible, namely to design the working parameters of airgun source reasonable. The other is using hydrophone array to improve spatial gain. 
The optimization measures can be changed according to the engineering implementation requirements.

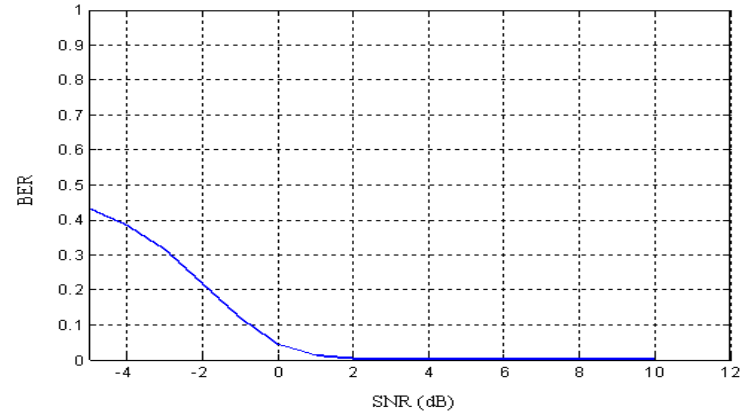

Fig. 3 BER curves under different SNR

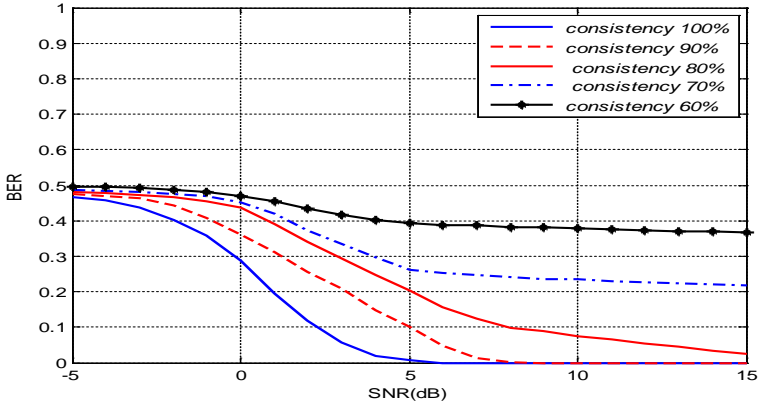

Fig. 4 BER curves to different pulse consistency

The pulse consistency is also one of the important factors which affect the performance of communication. According to the BER simulation and statistics of different pulse consistency in different SNR conditions as illustrated in Fig. 4, the higher the consistency is, the lower the BER of the communication is.

The multi-path effects largely to the performance of underwater acoustic communication, and it's difficult to eliminate. It is also the most common problem and the most difficult problem to overcome in underwater acoustic communication. This paper has done some simulations to study the way to eliminate multi-path effect, and finds that increasing the encoding interval can effectively eliminate the influence of multi-path. Under the same condition of the SNR and the ocean environment, communication BER decreases as the coding interval increases. The result suggests double the multi-path time as the encoding interval, so that the influence of multi-path can be reduced or eliminated.

In addition, continuous sending with multiple sources array can increase the time domain bandwidth product of the signal, improve the gain of matched filtering, so as to improve the detection probability of communication, reduce the BER of the communication system, and ensure the reliability and robustness of the system.

\section{Conclusion}

Airgun source can generate strong pulse with many advantages like high SL, low frequency and easy-coding, which is the performance advantage that traditional transducers do not have. This paper simulated and analyzed the feasibility of long-distance underwater acoustic communication using airgun source as the sound source, and proposed specific technical solutions for the pulse coding and detection. A new way is proposed to achieve long-distance underwater operation equipments. But performance under the real complex underwater acoustic channel conditions needs further experiment to verify.

\section{References}

[1] J. Tang: Study on Active Source Character and Weak Signal Detection In Reginal Scale Deep Exploration. PhD Dissertation, University of Science and Technology of China (2008), p. $38-40$

[2] A.D: The Measurement of Signals with Noise. Harbin: Publishing House of Harbin Engineering University (2001), p. 113-124

[3] Z.J.WANG: Research on Several Key Technologies of Underwater Acoustic Counter Measure. Harbin: A dissertation for degree of M.Eng of Harbin Engineering University (2010), p. 43-44

[4] S.J.ZHAO: Signal Detection and Estimation Theory. Beijing: Press of Tsinghua University (2005), p. 150-157 